Gynäk. Rdsch. 1985;25(suppl. 2):I-XII

\title{
Contents, Vol. 25, Supplement 2, 1985
}

\section{Inhalt}

Editorial XI

1. Hauptthema: Das Ovarialkarzinom

Hauptreferat

Pickel, H.; Lahousen, M.; Lichtenegger, W.; Stettner, H.: Die Bedeutung des Lymph-

knotenstatus für die Therapie des Ovarialkarzinoms 1

Koreferate

Pateisky, N.: Die Radioimmunszintigraphie, eine wertvolle Bereicherung im Rahmen der Diagnostik und Therapieplanung beim Ovarialkarzinom 5

Lichtenegger, W.; Pickel, H.; Lahousen, M.; Stettner, H.: Die operative Behandlung des Ovarialkarzinoms 8

Salzer, H.; Gitsch, E.; Sevelda, P.; Dittrich, Ch.; Karrer, K.; Österr. Gemeinschaft zur Therapie des Ovarialkarzinoms: Die stadiengerechte Therapie des Ovarialkar zinoms unter besonderer Berücksichtigung der Ergebnisse der 1. Österreichischen Ovarialkarzinomstudie 11

Lahousen, M.; Pickel, H.; Lichtenegger, W.; Stettner, H.: Ergebnisse der zytostatischen Chemotherapie beim Ovarialkarzinom 15

Mitteilungen zum 1. Hauptthema

Breitenecker, G.; Schieder, K.; Szepesi, T.; Nowotny, Ch.; Kölbl, H.; Janisch, H.:

Karzinome niedriger maligner Potenz (Borderline-Tumoren) des Ovars: Patho-

logie - Therapie - Prognose 20

Beck, A.; Rosen, A.; Baumgartner, G.: Die Ergebnisse der Behandlung des Ovarial karzinoms 1982-1984 22

Inhalt

IV

Bieglmayer, Ch.; Schieder, K.; Nowotny, Ch.; Kölbl, H.; Breitenecker, G.; Csaicsich, P.; Szepesi, T.; Janisch, H.: Biologische Heterogenität von Ovarialkarzinomen:

Rezeptor- und Tumorstammzellanalysen 24

Schieder, K.; Kölbl, H., Bieglmayer, Ch.; Csaicsich, P.; Nowotny, Ch.; Breitenecker, G.;

Szepesi, T.; Janisch, H.: Kann der Tumorstammzellassay einen Beitrag zur Therapieplanung bei

Patientinnen mit malignen Ovarialtumoren liefern? ... 27

Heidegger, H.; Fronius, G.; Nagel, W.; Krone, H.A.: Stellenwert der Sonographie und der Computertomographie in der Diagnostik benigner und maligner Ovarial tumoren 29

Nowotny, Ch.; Szepesi, T.; Janisch, H.; Breitenecker, G.; Dadak, Ch.; Kölbl, H.;

Schieder, K: Zum Stellenwert der postoperativen Chemotherapie mit Adriamycin/Cisplatin beim Stadium III des Ovarialkarzinoms überprüft an Hand von Second Look Operationen $(\mathrm{n}=28) \quad 32$

Christmann, D.; Strobel, E.: Therapieergebnisse mit dem PAC-Schema beim fortge- 
schrittenen Ovarialcarzinom 34

Fuith, L.C.; Hetzel, H.; Dapunt, O.: Chemotherapie von Ovarialkarzinomen mit Cisplatinum und Adriamycin 35

Dittrich, Ch.; Sevelda, P.; Salzer, H.: Second Line Chemotherapie bei Patientinnen mit fortgeschrittenem Ovarialkarzinom - Hexamethylmelamin, Vepesid und 5-Fluorouracil $\quad 37$

Lechner, W.; Reider, W.; Hetzel, H.; Daxenbichler, G.; Marth, Ch.: Zur Frage der Myocardschädigung unter Chemotherapie mit Epirubin-Cisplatin 39

Nowotny, Ch.; Janisch, H.; Führlinger, B.; Schieder, K; Micksche, M.: Intraperitoneale Immuntherapie mit OK-432 bei Patientinnen mit fortgeschrittenem Ova rialkarzinom und Aszites 40

Marth, Ch.; Mayer, I.; Gastl, G.; Schiechtl, J.; Rogan, A.; Daxenbichler, G.: Wachstumseffekte von Alpha-2 und Gamma-Interferon auf Ovarial- und Mammakarzinomzellen in vitro $\quad 42$

Szepesi, T.; Kärcher, K.H.; Stadler, B.; Schieder, K; Nowotny, Ch.; Kölbl, H.; Breitenecker, G.; Schlappack, O.; Janisch, H.; Dadak, Ch.: Postoperative Ganzabdomenbestrahlung mit Moving-Strip bei Ovarial-Karzinom: Grenzen, Technik, Nebenwirkungen 44

Tatra, G.: Untersuchungen über Akute-Phase-Reaktanden beim Ovarialkarzinom 46 Czerwenka, K; Rokitansky, A.; Buxbaum, P.; Trubel, W.; Hosmann, J.: Histochemischer Nachweis von Lektin-Bindungsstellen in Zystadenokarzinomen (des Ovars) im Vergleich zum Steroid-Rezeptorgehalt 47

Anderl, P.; Daxenbichler, G.; Fuith, L.C.; Marth, Ch.; Dapunt, O.: Korrelation zwischen Steroidhormonrezeptoren, histologischen und klinischen Parametern beim Ovarialkarzinom 49

Fuith, L.C.; Hetzel, H.; Daxenbichler, G.; Dapunt, O: Bestimmung des tumorassoziierten Antigens CA-125 im Serum von Patientinnen mit Ovarialkar zinomen 51

Sevelda, P.; Salzer, H.; Dittrich, Ch.; Pateisky, N.; Spona, J.: Die klinische Relevanz desneuen TumormarkersCA-125 53

Kölbl, H.; Schieder, K.; Bieglmayer, Ch.; Nowotny, Ch.; Csaicsich, P.; Szepesi, T.; Breitenecker, G.; Janisch, H.: CA-125, ein Parameter zur Verlaufskontrolle bei Patientinnen mit Ovarialkarzinom 55 Inhalt

IX

Ralph, G.; Motter, W.; Auner, H.; Lichtenegger, W.: Terminüberschreitung - ist eine Geburtseinleitung indiziert? 251

Egarter, Ch.; Kofler, E.; Husslein, P.: Vergleich zwischen aktivem versus expektativem Verhalten am Geburtstermin 253

Motter, W.; Ralph, G.; Lichtenegger, W.: Auswirkungen der intravaginalen Applikation von Prostaglandin-Ei-Tabletten auf Mutter und Kind im Rahmen der programmierten Geburt 256

Schausberger, L.; Moser, W.; Golob, E.: Geburtseinleitung mit Prostaglandin E2 Vaginaltabletten 257 
Wachter, I.; Weissenbacher, E.R.; Jany, J.; Gutschow, K.; Schneider, A.: Prospektive Untersuchungen zur Veränderung der aeroben Keimflora in Schwangerschaft und Wochenbett 259

Zeichen, H.-L.; Reiterer, F.; Rosanelli, K.; Rosegger, H.: Sonographische Befunde bei reifen Neugeborenen nach Zangengeburt 260

Beck, A.; Vutuc, Ch.: Eine Analyse indirekter Müttersterbefalle in Österreich .... 263

Knitza, R.; Wisser, J.; Mempel, M.: Idiopathische thrombozytopenische Purpura und Schwangerschaft 265

Langer, M.; Ringler, M.: Zur Didaktik des geburtshilflichen Internates: Kontinuierliche Geburtsbegleitung durch Studenten 267

Neumann, H.: Schwangerenbetreuung in der Praxis: Erfolge und Schwierigkeiten . . 269

Wisser, J.; Bald, R.; Knitza, R.: Indikationen zur vorzeitigen Beendigung der

Schwangerschaft nach Diagnostik lebensfähiger fetaler Entwicklungsstörungen 271

Fronius, G.; Heidegger, H.: Placentamo hologie dargestellt im Histogramm .... 273

Steldinger, R.; Deichel, A.; Jacob, S.: Schwangerschaft und Geburt bei Epilepsie . . 275

Reinthaller, A.; Deutinger, J.; Miiller-Tyl, E.; Riss, P.; Fischl, F.; Neumark, J.;

Janisch, H.: Bestimmungen schwangerschaftsspezifischer Parameter in der Früh-

schwangerschaft und deren Wertigkeit für die Prognose des Schwangerschafts-

verlaufes im Rahmen eines in vitro Fertilisierungsprogrammes $\quad 278$

Autorenregister

281

Inhalt

$\mathrm{V}$

Schröck, R.; Graeff, H.; Hafter, R.; Schmid, L.: Differenzierte Verlaufsbeurteilung fortgeschrittener Ovarialkarzinome mittels tumorassoziierter Antigene .... 58

Urdl, W.; Desoye, G.; Pickel, H.; Lahousen, M.: Der immunhistochemische Nachweis von CEA und anderen Tumormarkern im Gewebe von Ovarialkarzinomen 60

Kaesemann, H.; Paulick, R.;Caffier, H.: Bedeutung sogenannter Tumormarker (BKS, CEA, PHI, TPA, CA-125) in der Verlaufskontrolle des Ovarialkarzinoms 62

2. Hauptthema: Uberwachung der Austreibungsperiode mit CTG und MBU

Hauptreferat

Künzel, W.: Die biochemische Kontrolle als Alternative zur Uberwachung des Feten

während der Austreibungsperiode? 65

Koreferat

Dapunt, O.: Uberwachung der Austreibungsperiode mit CTG und MBU

81

Mitteilungen zum 2. Hauptthema

Walcher, W.; Lichtenegger, W.; Tscherne, K.; Auner, H.; Haas, J.; Reif, R.: Vergleich

von CTG und Mikroblutuntersuchung in der Austreibungsperiode mit dem

fetal outcome 88

Pfersmann, Ch.; Gruber, W.; Endler, M.: Die Austreibungsperiode nach Epiduralanästhesie 90

Dadak, Ch.; Neunteufel, W.; Gring, H.; Janisch, H.: Die Austreibungsperiode und das fetal outcome der Neugeborenen unter $2500 \mathrm{~g} 92$

Rudelstorfer, R.; Simbruner, G.; Bernaschek, G.; Janisch, H.: Blutuntersuchungen und Wärmeflußmessungen vom fetalen Scalp: Vergleichbare Überwachungs-

methoden in der Austreibungsperiode? 
Stummvoll, W.; Nagl, F.; Prestel, A.; Wiebogen, L.: Das interne CTG der Austrei bungsperiode von über 5000 Geburten 96

Prestel, A.; Nagl, F.; Stummvoll, W.; Wiebogen, L.: Der diagnostische Wert der internen Tokometrie 98

Hägele, D.; Berg, D.: Das CTG während der Sectio 99

Schlotter, CM.: Fetale Herzfrequenzmuster und Azidose-Risiko der Austreibungs periode $\quad 100$

3. Hauptthema: Fruchtwasserdiagnostik und Chorionbiopsie

Hauptreferat

Brusis, E.: Fruchtwasserdiagnostik und Chorionbiopsie 103

Koreferat

Huber, J.C.; Müller, M.M.; Spona, J.; Wagenbichler, P.: Methodik, erste Erfahrungen und Möglichkeiten der molekularbiologischen pränatalen Diagnostik

112

Inhalt

VI

Mitteilungen zum 3. Hauptthema

Reichel, R.; Karlic, H.; Czerwenka, K.; Huber, J.C.: Eine immunhistochemische Methode zur Differenzierung fetaler Trophoblastzellen von mütterlichen Zellen 118

Hofmann, H.M.H.; Zierler, H.; Brunner, S.; Rosenkranz, W.; Kainer, F.: Die Cho-

rionbiopsie als Alternative zur Amniozentese? 120

Zahn, F.W.: Pranatale Diagnostik in einer gynäkologischen Praxis 122

Popp, L.W.; Müller-Holve, W.; Stoeckenius, M.; Martin, K.: Verschiedene Methoden der Materialgewinnung vom Chorion: Zytologiebürstenmethode, Aspirations-

biopsie, Chorionskopie - erste Erfahrungen und Vergleich 123

Bartosch, B.; Schnedl, W.; Haden, H.: Pranatale Diagnostik mittels CVS durch Biopsie-Katheter und Biopsie-Zange 126

Genger, H.; Schnedl, W.; Scholz, P.; Wagenbichler, P.: Pranatale Diagnose durch

Amniozentese oder Chorionbiopsie 128

Kainer, F.; Hofmann, H.M.H.; Winter, R.; Brunner, S.; Rosenkranz, W.; Zierler, H.:

Pranatale Diagnostik genetischer Defekte mittels Amniozentese: Erfahrung

über 2500 Punktionen 130

Gloning, K.-Ph.; Brusis, E.; Bauer, S.; Döring, K.: Schwangerschañen mit und ohne

Frühamniozentese: Verlaufund Befunde bei den Neugeborenen 132

Rempen, A.; Feige, A.; Osterhage, H.R.; Fiedler, K.: Harnwegsfehlbildungen - prana tale Diagnostik und postpartale Ergebnisse 134

Brackertz, M; Feige, A.: Präkonzeptionelle Strahlenexposition beider Elternteile als mögliche Ursache einer pränatal diagnostizierten de novo entstandenen kom-

plexen strukturellen Chromosomenaberration

Sarellitenprogramm der Firma CILAG GmbH

Hugo, R. v.; Lenz, A.; Hafter, R.: Veränderungen der Blutgerinnung bei hormonaler

Kontrazeption 138

Freie Vorträge: Gynäkologie

Hosmann, J.; Grünberger, W,; Söltz-Szöts, J.; Poitschek, Ch.: Mykoplasmen bei prämenarchalen Kindern (eine vaginoskopische Studie) 142

Popp, L.W.; Müller-Holve, W.; Martin, K.: Vaginosonographie mit dem $7 \mathrm{MHz}$

Fingertipscanner - erste Erfahrungen 144 
Gutschow, K; Weissenbacher, E.R.; Adam, D.; Schneider, A.; Wachter, J.; Lühr, H.G.: Serum- und Gewebekonzentrationen von Ciprofloxacin bei gynäkologi schen Infektionen 146

Gerstner, G.J.; Müller, G.; Kronich, W.; Dalhoff, A.; Weuta, H.: Pharmakokinetik von Ciprofloxacin (BAY o 9867) in Uterus und Adnexen 149

Mayer, H.O.; Auner, H.: Ergebnisse mikrobiologischer Untersuchungen bei Fluor vaginalis 151

Wiedemann, R.; Scheidel, P.; Hepp, H.: Untersuchungen an der Hydrosalpinx morphologische und biophysikalische Aspekte 153

Mayer, H.O.; Brunner, J.; Hofmann, H.M.H.; Ralph, G.; Schweditsch, M.O.: Sonographische Befunde bei Verdacht aufExtrauteringravidität 154

Feiks, A.; Dadak, Ch.; Deutinger, J.; Reinthaller, A.; Janisch, H.: Tubendurchgängigkeitsprüfung nach konservierender Operation von Tubargraviditäten 156 Inhalt

VII

Pateisky, N.; Philipp, K.; Kubista, E.; Hamilton, G.; Czerwenka, K.: Der Stellenwert der Immunszintigraphie im Rahmen des präoperativen Lymphknotenstagings beim Mammakarzinom 158

Schröcksnadel, H.; Haid, Ch.; Heim, K.; Martin, J.: Ansätze zur Verbesserung der Mammasonographie mit Hilfe hochfrequenter nahfokussierter Schallköpfe 160

Hofmann, H.M.H.; Lahousen, M.; Schneeweiß, W.D.: Friiherkennung des Endometriumkarzinoms mit Abradul 162

Lahousen, M.; Schneeweiß, W.D.; Zechner, M.: Prognostische Faktoren beim Endometriumkarzinom des Stadium I 164

Lahousen, M.: Lymphadenektomie beim Korpuskarzinom 166

Lahousen, M.; Pickel, H.; Urdl, W.; Pürstner, P.; Beaufort, F.: Die Aussagekraft einer Kombination von Tumormarkern in der gynäkologischen Onkologie 168 Albrich, W; Götz, A.; Stein, G.; Schubert-Fritschle, G.; Waidelich, W.: Die Behandlung des Vulvakarzinoms an der Frauenklinik der Universität München im Klinikum Großhadern von 1973 bis $1984 \quad 171$

Bieglmayer, Ch.; Breitenecker, G.; Schieder, K.; Lunzer, E.: Immunhistochemische Darstellung von Basalmembranen mittels eines monoklonalen Antikörpers gegen Typ IV Kollagen 174

Schüßler, B.; Philipp, R.; Lentsch, P.: Pathogenese und Therapie postoperativer Blasenentleerungsstörungen in der Gynäkologie $\quad 178$ Hetzenauer, A.; Bazzanella, A.; Reider, W.: Zur Inzidenz und Bedeutung der instabilen Urethra 180

Grünberger, W.; Freilinger, G.: Die Insellappen-Plastik: Eine neue Kolpoperineoplastik bei engem Introitus und/oder Vaginalstenose 182

Grünberger, W.: Neovagina nach Vecchietti - teilweise modifizierte Operationstechnik und Nachbehandlung 183

Schröck, R.; Steuer, G.; Hölscher, M.: Extraovarieller maligner Brenner-Tumor: Fallbericht und Deutung 185

Vutuc, Ch.; Breitenecker, G.: Kohortenanalyse der Zervixkarzinomsterblichkeit in Österreich $\quad .187$ 
Gredler, B.; Gerstner, G.: Angewandte Friiherkennung des Zervixkarzinoms auf regionaler Primärversorgungsebene - Modellprojekt „Korneuburg/Stockerau” 189 Schneeweiß, W.D.: Kolposkopische Bilder und ihre histologischen Grundlagen ... 190 Ralph, G.; Kainer, F.: Ergebnisse urodynamischer Untersuchungen nach derabdominalen Radikaloperation des Zervixkrebses 192

Hosmann, J.; Laufer, G.; Czerwenka, K.; Wollenek, G.; Reinold, E.: Der NeodymYAG Laser - Wirkung und Regeneration am Portiogewebe 194

Hötzinger, H.; Wischnik, A.: Rektosonographie und ihre Bedeutung in der Diagnose und Therapie postoperativer Lokalrezidive gynäkologischer Tumoren 196 Lahousen, M.; Pickel, H.: Chemotherapie beim fortgeschrittenen bzw. rezidivierenden Zervixkarzinom 198

Lasnik, E.; Tatra, G.: Kommt es durch die primäre Radiatio beim Zervixkarzinom zu einer Veränderung des Sexuallebens? 200

Stummvoll, W.; Brandtner, W.: Einsatz des Computertomographen zur radiologischen Beckenmessung 201

Mutke, H.G.: Juristische Probleme bei der heterologen Insemination (AID)

Inhalt

VIII

Metka, M.; Haromy, T.; Huber, J.C.; Schurz, B.; Kozak, W.: Apparative Insemina tion unter dem Mikromanipulator 205

Szalay, S.; Spernol, R.; Nachtigall, M: Ein Jahr in vitro Fertilisierung in Klagenfurt Ergebnisse und Erfahrungen 207

Metka, M.; Karlic, H.; Huber, J.C.: Erfahrungen mit dem Hamsterei-Spermien-Penetrationstest in der Sterilitätsroutine 208

Hosmann, J.; Vytiska-Binstorfer, E.; Huber, J.C.; Söltz-Szöts, J.: Inzidenz bakterieller Genitalinfektionen bei Sterilitätspatientinnen 211

Huber, J.C.; Schurz, B.; Metka, M.; Knogler, W.; Spona, J.: Die erfolgreiche thyreoidale Therapie einzelner Formen hypergonadotroper Amenorrhoen 213

Schurz, B.; Schmidt, J.B.; Huber, J.C.: TRH-Test bei Frauen mit sekundärer Amenorrhoe und Hirsutismus 214

Schurz, B.; Huber, J.C.; Knogler, W.; Weindlmayr, M.: Veränderung im Beta-Endorphinspiegel unter einer Gonadotropin-Releasing-Hormonbehandlung 217 Langer, M.; Huber, J.C.; Knogler, W.; Spona, J.: Lutealanalytische Rezeptorenbestimmung 220

Bieglmayer, Ch.; Fischl, F.; Müller-Tyl, E.: Der HI-Estrotec-Test - eine neue immunologische Methode zur Östrogenbestimmung im Harn 222

Freie Vorträge: Geburtshilfe

Mutke, H.G.: Timing-Störungen zwischen Mutter und Kind und die „natürliche” Geburt? 225

Bieglmayer, Ch.; Rudelstorfer, R.; Bartl, W.; Janisch, H.: In vitro Adsorption zirkulierender Immunkomplexe an plazentares Basalmembrankollagen bei Gestose 227 Rudelstorfer, R.; Simbruner, G.; Tabsh, K.; Khoury, A.; Wasserstrum, N.; Janisch, H.; Assali, N.S.: Ritodrine Infusion - Auswirkungen auf Sauerstoffverbrauch und Wärmefluß der utero-fetoplazentaren Einheit 229

Rammer, E.; Friedrich, F.: Klinische Relevanz der Östriol-Bestimmung in der Schwangerschaft 231 
Kowatsch, A.W.: Bipolare Reizstromdiagnostik als Indikator der Wehenbereitschaft 233

Girardi, F.; Mayer, H.O.; Sperger, H.; Haas, J.: Das Stützpessar - eine Therapiemöglichkeit bei vorzeitiger Eröffhung des Verschlußapparates 236

Wischnik, A.; Weidenbach, A.: Neuere Konzepte fur die intravenöse und orale Tokolyse 238

Vytiska-Binstorfer, E.; Salzer, H.; Simbruner, G.: Ambroxol im Vergleich zu Betamethason zur Förderung der antepartalen Lungenreife - eine klinische Doppel-

blindstudie 241

Auner, H.; Ralph, G.; Lichtenegger, W.; Mayer, O.; Walcher, W.; Schweditsch, M.O.:

Bedeutung immunologischer und enzymatischer Marker für das geburtshilfliche

Vorgehen beim vorzeitigen Blasensprung 243

Weiss, P.A.M.; Hofmann, H.M.H.: Fruchtwasserdiagnostik bei Gestationsdiabetes . 247

Schweditsch, M.O.; Desoye, G.; Kostner, G.M.; Pfeiffer, K.H.; Zechner, R.: Lipide, Lipoproteine, Plazentahormone und Insulin während der Gravidität 248

Fuchs, G.; Rost, I.; Leodolter, S.; Marhold, W.: Komplikationen in der Gravidität bei Diabetikerinnen

249

\section{Editorial}

Wie jedes zweite Jahr, findet die Jahrestagung der Österreichischen Gesellschaft für Gynäkologie und Geburtshilfe gemeinsam mit der Baye-rischen Gesellschaft für Geburtshilfe und Frauenheilkunde statt. Diese gemeinsamen Tagungen sind immer von besonderem Reiz: es kommt so zu belebenden Konfrontationen der Ansichten, des Vorgehens und der Erfolge unserer fachärztlichen Tätigkeit.

Wie seit langem bewährt, wurde auch diesmal das wissenschaftliche Programm von den Präsidenten der beiden Gesellschaften, Herrn Prof. Dr. F. Zimmer aus München-Pasing und Herrn Prof. Dr. E. Burghardt als Tagungspräsident, gemeinsam erstellt, tatkräftig unterstützt von Herrn Doz. Dr. W. Lichtenegger als Tagungssekretär.

Die 3 Hauptthemen waren von großer Aktualität, es wurde jeweils sowohl von deutschen als auch österreichischen Kollegen referiert. Dem umfangreichen 1. Hauptthema, dem „Ovarialkarzinom”, wurden 5 Refe-rate und zahlreiche Vorträge zum Thema gewidmet. Das 2. Hauptthema hieß „Überwachung der Austreibungsperiode mit CTG und MBU”, das durch Refer at und Koreferat, sowie ebenfalls zahlreichen themenbezoge-nen Vorträgen behandelt wurde. Ähnlich wurde das 3. Hauptthema abgehandelt, das der aktuellen „Fruchtwasserdiagnostik und Chorion-biopsie" gewidmet war. Es wurde so ein geschlossener Überblick des gegenwärtigen Standes auf diesem Gebiet gegeben. Die sogenannten „Freien Vorträge” aus dem Bereich der Gynäkologie und Geburtshilfe waren nicht themenbezogen und gaben eine gute Ubersicht über unser gesamtes Fachgebiet.

Editorial

XII

Die Herstellung des Kongreß-Supplementes ist immer mit beträchtli-chen Kosten verbunden. Auch in diesem Jahr gilt unser besonderer Dank jenen österreichischen Firmen, die, als Sponsoren, das Erscheinen ermög-licht und damit ihre Verbundenheit mit unserem Fachgebiet bewiesen haben. 
Unser Dank gilt aber auch den Mitarbeitern des Verlages S. Karger, Basel, und der Druckerei Ferdinand Berger, Horn in Niederösterreich, für die gute Zusammenarbeit, um die kurzfristige Herstellung des Bandes zu gewährleisten.

E. Gitsch E. Reinold 